\title{
BREATHING SOUNDS - DETERMINATION OF EXTREMELY LOW SPL
}

\author{
M. Harun ${ }^{1 *}$, R. Teoh Y. S ${ }^{1}$, M. 'A. A. Ahmad, M. Mohd. Mokji', You K. Y' ${ }^{1}$, S. A. \\ R. Syed Abu Bakar ${ }^{1}$, P. I. Khalid ${ }^{1}$, S. Z. Abd. Hamid ${ }^{1}$ and R. Arsat ${ }^{1}$ \\ ${ }^{1}$ School of Electrical Engineering, \\ Universiti Teknologi Malaysia, \\ *Email: mokhtarharun@utm.my \\ Phone: +6075535358
}

\begin{abstract}
Breathing sound is an extremely low SPL that results from inspiration and expiration process in the lung. Breathing sound can be used to diagnose persons with complications with breathing. Also, the sound can indicate the effectiveness of treatment of lung disease such as asthma. The purpose of this study was to identify SPL of breathing sounds, over six one octave center frequencies from $63 \mathrm{~Hz}$ to $4000 \mathrm{~Hz}$, from the recorded breathing sounds in wav files. Breathing sounds of twenty participants with normal weight BMI had been recorded in an audiometry room. The breathing sound was acquired in two states: at rest and after a 300 meters walk. Matlab had been used to process the breathing sounds that are in .wav files to come up with SPL (in $\mathrm{dB}$ ). It has been found out that the SPL of breathing sound of all participants are positive at frequencies $63 \mathrm{~Hz}$ and $125 \mathrm{~Hz}$. On the other hand, the SPL are all negatives at frequency $1000 \mathrm{~Hz}, 2000 \mathrm{~Hz}$ and $4000 \mathrm{~Hz}$. In conclusion, SPL of breathing sounds of the participants, at frequencies $250 \mathrm{~Hz}$ and $500 \mathrm{~Hz}$ that have both positive and negative values are viable to be studied further for physiological and medicinal clues.
\end{abstract}

Keywords: Sound pressure, extremely low SPL, respiratory, breathing sound.

\section{INTRODUCTION}

Measurement of the extremely low sound pressure level (SPL) is important to evaluate acoustics environment such as audiometry room and recording studios. Noise emitted from quiet machinery and equipment such as lighting armatures is also part of extremely low SPL [1]. Elements in extremely low SPL in human such as breathing sound and heart sound are important to determine characteristics of physiological qualities for monitoring of organ functioning, effectiveness of respiratory treatment, and detection of disease [2][3].

By using compact and conventional sound level meter (SLM), direct measurement of extremely low SPL using sound level meter is very challenging due to two factors. First, the reference sound pressure which is $20 \mu \mathrm{Pa}$ used in the meter is the lowest sound pressure the machine can detect. Then, the meter will give the reading 0 $\mathrm{dB}$. If the impinging sound pressure on the microphone is lower than $20 \mu \mathrm{Pa}$, these sounds will not show up on the display on the meter. Second, the inherent noise in the SLM adds up the level of the noise is to be measured. Even if the noise level is known 
and compensated for, a satisfying measurement can only be done for levels $2-5 \mathrm{~dB}$ above noise floor [4].

The low frequency SPL causes annoyance even if it is below the limiting value, typically, $30 \mathrm{~dB}$. For the measurement of low frequency extremely low SPL, the operational high pass filter has to be set to $20 \mathrm{~Hz}$, so as not to overload the meter due to probable high sound pressure of low frequency noise input (Bjor, 1999). The measurement of low SPL at low frequency incurs high bias error and large scatter, Bias error indicates how the data deviate from the data average value. Large scatter in the data set will cause poor reproducibility. From the measurement of low sound pressure levels at low frequencies conducted in an Inter Nordic Round Robin comparison with 5 laboratories, it has been found out that the low frequency noise level has poor reproducibility up until frequency $200 \mathrm{~Hz}$. The reproducibility is in the higher order of $15 \mathrm{~dB}$. At frequency $250 \mathrm{~Hz}$ and higher, the bias error has gone down and reproducibility has improved satisfactorily. The findings also assert that noise due to handling of measurement instrument must be $6 \mathrm{~dB}$ below the measurement signals [4].

Breathing is also called ventilation or respiration is an act that consists of two breaths. One breath that is called inspiration is the process to take air into the lungs. The other breath, which is called expiration, is the process to take air out of the lungs. Ineffective ventilation can negatively impact efficacious gas exchange. For an adult with healthy lungs (or non-respiratory compromised), number of breathing should be normal between 12-20 number of breath per minute [[5] and [6], as cited in [3]]. In hospital, respiratory rates are used as the early warning scores (EWS) vital signs. When the breathing rate slows down to 8 breaths per minute or less, a patient is about 18 times higher odds of death within 24 hours when compared to a patient with a normal respiratory rate [[7], as cited in [3]].

Breathing sounds are extremely low SPL that occurs mostly at low frequency (Figure 1). A woman has breathing sounds at higher frequency compared to man [9]. Breathing complication sound such as wheezing sounds have pitch at $100 \mathrm{~Hz}$ and 1000 Hz. Rhonchi sounds, which is similar to wheezes but coarser has pitch around $150 \mathrm{~Hz}$ [8].

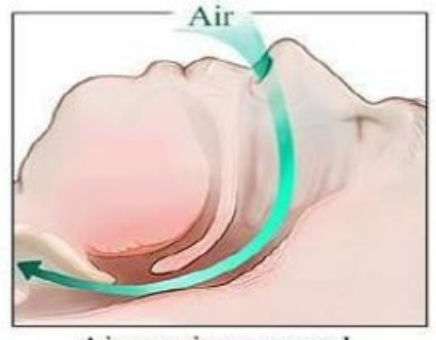

Airway is open and air moves through

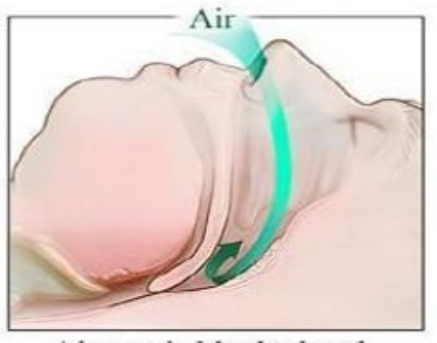

Airway is blocked and air does not move through

Figure 1. Breathing process: Normal (left); Obstructive breathing (right) [8] 


\section{METHODOLOGY}

\section{Experimental Setup}

Twenty fourth-year students from School of Electrical Engineering (SEE) UTM Skudai had participated in the study. They were 10 males and 10 females. They shared the common BMI which is normal weight, in the range of BMI 20-25. The students were given specified time and date to come to the laboratory for the study. Audiometry room in Acoustics Research Laboratory, SEE UTM Skudai had been used as recording room. RealWave sound recorder that comes with $50 \mathrm{mV} / \mathrm{Pa}$ sensitivity microphone had been used to record the breathing sounds. The breathing sounds from each participant were recorded in two states: at rest and after 300 meters walk. For breathing sound recording, at rest, the participants was let resting in the lab not fewer than 5 minutes before the breathing sound was recorded. Then, the participants were asked to walk about 300 meters along the corridors of school building. After the participant finished the walk, breathing sound of the participant was recorded once again. The recorded breathing sounds (in .wav files) were compiled in terms of participants ID, gender, and breathing states: Rests or Walk. Matlab had been used to analyse these .wav files to output SPL at their respective frequency.

\section{RESULTS AND DISCUSSION}

Table 1 show SPL at one-octave frequency $63 \mathrm{~Hz}$ to $400 \mathrm{~Hz}$ breathing sounds for male participant at rest. SPLs at one-octave frequency $63 \mathrm{~Hz}$ and $125 \mathrm{~Hz}$ are all positives indicating that the sound pressures at these two frequencies are all above the reference pressure of $20 \mu \mathrm{Pa}$. Beginning at frequency $250 \mathrm{~Hz}$ up until $4000 \mathrm{~Hz}$, more SPL of breathing sound that is below $20 \mu \mathrm{Pa}$ has been found from the participants' recorded breathing sounds. At frequencies $2000 \mathrm{~Hz}$ and $4000 \mathrm{~Hz}$, the measured SPL of breathing sound of male participants at rest are all negatives (sound pressure below $20 \mu \mathrm{Pa}$ ).

Table 1. SPL (in dB) versus frequency $(\mathrm{Hz})$ of sampled data - Male (Rest).

\begin{tabular}{cccccccc}
\hline $\begin{array}{c}\text { Participant/ } \\
\begin{array}{c}\text { Frequency } \\
(\mathrm{Hz})\end{array}\end{array}$ & 63 & 125 & 250 & 500 & 1000 & 2000 & 4000 \\
\hline 1 & 25 & 29 & 16 & 1 & -13 & -11 & -13 \\
2 & 26 & 26 & 21 & 9 & -5 & -10 & -3 \\
3 & 26 & 26 & 14 & 7 & -10 & -2 & 0 \\
4 & 23 & 12 & 0 & -4 & -9 & -6 & -7 \\
5 & 28 & 10 & 9 & 0 & -14 & -7 & -9 \\
6 & 35 & 28 & 20 & 7 & -5 & -14 & -1 \\
7 & 8 & 19 & -1 & 0 & -12 & -19 & -17 \\
8 & 30 & 15 & 6 & 6 & 1 & -12 & -5 \\
9 & 31 & 19 & 21 & -1 & -5 & -10 & -10 \\
10 & 15 & 19 & 18 & 5 & -27 & -11 & -12 \\
\hline
\end{tabular}


It is apparent from Figure 2 that the SPL of breathing sound conforms to the conventional equal loudness contour. If one to compare SPL value of any soft physiological sound from human such as snoring and sneezing, the difference of breathing sound is that its SPL are all negatives at frequency $2000 \mathrm{~Hz}$ and $4000 \mathrm{~Hz}$.

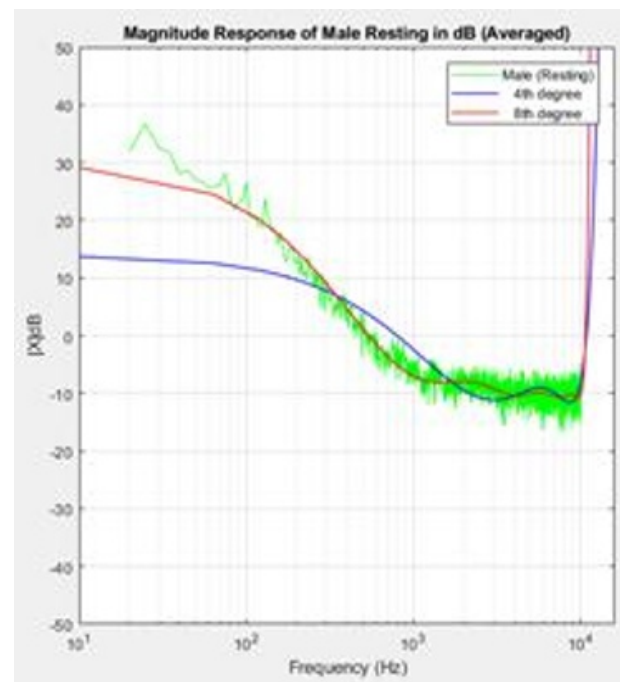

(a)

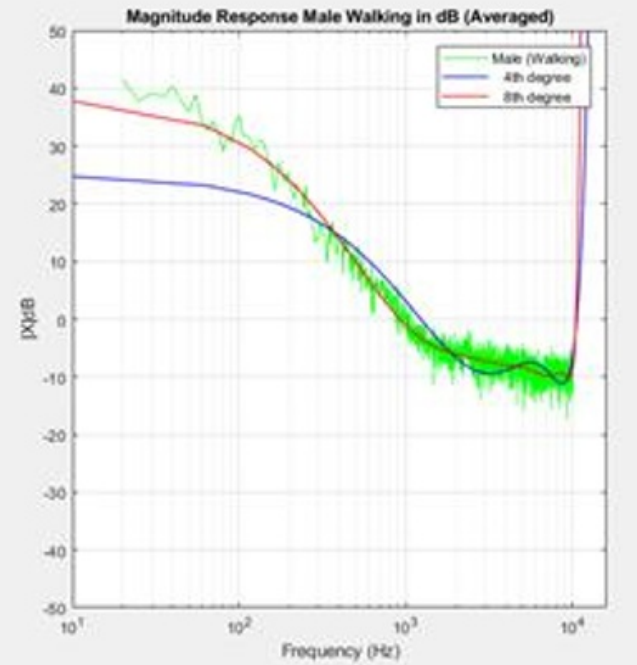

(b)

Figure 1: SPL (dB) versus frequency (Hz) for male: (a) Rest, (b) Walk.

\section{Statistical tests of the SPL results.}

From Table 2, it is found that the mean values of SPL of breathing sounds after a walk are generally higher at all frequencies for both male and female participants. The values of standard deviation are also consistent of the increase for both male and female participants. Therefore, the data confirms to the hypothesis that SPL of breathing sounds is higher when there is physical activities of the participants, and thus the rate of metabolism.

However, it is found (in Table 3) that the variance of the SPL data across all frequencies for female participants is quite large. It can be inferred that there exist many large SPL or outliers in the data of the ten female participants. Also in SPL data of female participants, except for the frequencies $250 \mathrm{~Hz}$ and $1000 \mathrm{~Hz}$, variances of the SPL of breathing sound is doubled for the Walk compared to the Rest.

Finally, from statistical significance tests between the Rest and the Walk, it is found that the SPL breathing sound for female participants is significance at frequencies $63 \mathrm{~Hz}, 125 \mathrm{~Hz}$, and $1000 \mathrm{~Hz}$. 
Table 2. Statistical tests of SPL (dB) - Descriptive

\begin{tabular}{cccccccccc}
\hline $\begin{array}{c}\text { Breath. } \\
\text { state }\end{array}$ & $\begin{array}{c}\text { Gender/ } \\
\text { Freq. } \\
\text { (Hz) }\end{array}$ & 63 & 125 & 250 & 500 & 1000 & 2000 & 4000 & Tests \\
\hline & $\mathrm{M}$ & $25(36)$ & $20(30)$ & $12(20)$ & $3(9)$ & $-10(-2)$ & $-10(-6)$ & $-8(-6)$ & Mean \\
& $\mathrm{F}$ & $28(33)$ & $25(34)$ & $12(21)$ & $3(10)$ & $-4(3)$ & $-9(-5)$ & $-13(-9)$ & \\
& $\mathrm{M}$ & $26(36)$ & $19(33)$ & $15(19)$ & $3(10)$ & $-9(0)$ & $-10(-7)$ & $-8(-5)$ & Med. \\
$\begin{array}{c}\text { Rest } \\
\text { (Walk) }\end{array}$ & $\mathrm{F}$ & $25(31)$ & $23(32)$ & $9(24)$ & $2(8)$ & $-5(-2)$ & $-8(-8)$ & $-13(-7)$ & \\
& $\mathrm{M}$ & $63(166)$ & $46(126)$ & $71(129)$ & $18(134)$ & $56(57)$ & $22(69)$ & $29(51)$ & Var. \\
& $\mathrm{F}$ & $72(294)$ & $114(216)$ & $143(163)$ & $115(331)$ & $66(163)$ & $24(165)$ & $32(12)$ & \\
& $\mathrm{M}$ & $8(13)$ & $7(11)$ & $8(11)$ & $4(12)$ & $7(8)$ & $5(8)$ & $5(7)$ & Std. \\
& $\mathrm{F}$ & $8(17)$ & $11(15)$ & $12(13)$ & $11(18)$ & $8(13)$ & $5(13)$ & $6(3)$ & $\mathrm{dev}$ \\
\hline
\end{tabular}

Note: $\mathrm{M}$ denotes male, and $\mathrm{F}$ is for female

Table 3. Statistical test of SPL (dB) - Significance

\begin{tabular}{ccccccccc}
\hline $\begin{array}{c}\text { Test of } \\
\text { significance }\end{array}$ & $\begin{array}{c}\text { Gender/ } \\
\text { Frequency } \\
\text { (Hz) }\end{array}$ & 63 & 125 & 250 & 500 & 1000 & 2000 & 4000 \\
\hline Significance & M & 0.41 & 0.13 & 0.12 & 0.34 & 0.14 & 0.35 & 0.05 \\
$(\mathrm{p}<0.05)$ & $\mathrm{F}$ & 0.04 & 0.03 & 0.12 & 0.15 & 0.04 & 0.22 & 0.68 \\
& $\mathrm{M}$ & No & No & No & No & No & No & No \\
Verdict & F & Yes & Yes & No & No & Yes & No & No \\
\hline
\end{tabular}

Note: $\mathrm{M}$ denotes male, and $\mathrm{F}$ is for female

\section{CONCLUSION}

From the study, it can be concluded that SPL data at $250 \mathrm{~Hz}$ and $500 \mathrm{~Hz}$ during Rest and Walk breathing states for both genders can be investigated further for clues because at these frequencies there exist both positive and negative SPLs. High difference of variance of SPL data between the Rest and the Walk for female participants has inferred that the data need to be investigated for the influence of noise. If the influence of noise can be ruled out, the acquired data might be able to shed light on SPL of breathing sound of women with respect to physical activities. Finally, it can be concluded that physical activities of participants with common BMI affect SPL of their breathing sounds at certain frequencies.

\section{ACKNOWLEDGEMENTS}

The authors would like to acknowledge Universiti Teknologi Malaysia and Kementerian Pengajian Tinggi for providing the financial assistance under research grants FRGS Vot 4F870 and GUP Vot 14J47. 


\section{REFERENCES}

[1] Bjor, Ole-H. (1999). Application note: Measurement of Extremely Low Sound Pressure Levels Ole-Herman Bjor, M.Sc. Norsonic AS. AN Low-noise mic. Ed. 1. Rev. 0 ENGLISH 04.99.

[2] Lozano-Garcia, M., Fiz, J. A., Martinez-Rivera, C., Torrents, A., Ruiz-Manzano, J., Jane, Â. R. (2017). Novel approach to continuous adventitious respiratory sound analysis for the assessment of bronchodilator response. PLoS ONE 12(2): e0171455. doi:10.1371/journal.pone.0171455.

[3] Flenady, T., Dwyer, T., Applegarth, J. (2017). Accurate respiratory rates count: So should you! Australasian Emergency Nursing Journal. Vol. 20 (2017), 45-47.

[4] Simmons, C. (2009). Measurement of sound pressure levels at low frequencies in rooms. NT Technical Report. TR385 (1997). Nordic Innovation Center (Norden). Oslo, Norway.

[5] Walsh M, Erb G, Kozier B. (2010). Fundamentals of Nursing: Frenchs Forest. N.S.W: Pearson Education; 21.

[6] McCance K. L, Huether S. E. (2015). Pathophysiology The biologic basis for disease in adults and children. Elsevier Health Sciences.

[7] Ljunggren M, Castrén M, Nordberg M, Kurland L. (2016). The association between vital signs and mortality in a retrospective cohort study of an unselected emergency department population. Scand J Trauma Resusc Emerg Med. Vol. 24(1): $21-$

[8] SASA - Scottish Association of Sleep Apnea. (2018). Website: https://scottishsleepapnoea.co.uk/patient-information/symptoms-diagnosis-andtreatment-of-sleep-apnoea/

[9] Gavriely, N., Nissan, M., Rubin, A. H., Cugell, D. W. (1995). Spectral characteristics of chest wall breathe sounds in normal subjects. Thorax 1995 50: 1292-1300. doi: 10.1136/thx.50.12.1292 